\title{
Modeling and analysis of taeniasis and cysticercosis transmission dynamics in humans, pigs and cattle
}

\author{
Joshua A. Mwasunda ${ }^{*} \mathbb{D}$, Jacob I. Irunde ${ }^{2}$, Damian Kajunguri ${ }^{3}$ and Dmitry Kuznetsov ${ }^{1}$
}

\section{"Correspondence:}

joshuamwasunda@gmail.com;

mwasundaj@nm-aist.ac.tz

'Department of Applied

Mathematics and Computational

Science, The Nelson Mandela

African Institution of Science and Technology, P.O. Box 447, Arusha,

Tanzania

Full list of author information is

available at the end of the article

\begin{abstract}
Taeniasis and cysticercosis pose a significant challenge to food safety and public health. Cysticercosis reduces the market value for pigs and cattle by making pork and beef unsafe for consumption. In this paper, a mathematical model for the transmission dynamics of taeniasis and cysticercosis in humans, pigs and cattle is formulated and analyzed. The analysis shows that both the disease free equilibrium (DFE) and the endemic equilibrium (EE) exist. To study the dynamics of the diseases, we derived the basic reproduction number $R_{0}$ by next generation matrix method. When $R_{0}<1$, the DFE is globally asymptotically stable whereas when $R_{0}>1$ the EE is globally asymptotically stable. The normalized forward sensitivity index was used to determine sensitive parameters to the diseases. Humans' recruitment rate, probability of humans' infection with taeniasis and the defecation rate of taenia eggs by humans with taeniasis are the most positive sensitive parameters to diseases' transmission whereas the human natural death rate is the most negative sensitive parameter. However, it is biologically unethical and not practical to increase human natural mortality rate for disease control. In this case, other parameters with negative sensitivity indices such as death rate of taenia eggs and proportions of unconsumed infected beef and pork can be considered for disease control. Generally, to control the diseases, more efforts should be made directed to reducing the number of humans who have taeniasis and defecate in the open environment. Also meat inspection and indoor keeping of cattle and pigs should be emphasized.
\end{abstract}

Keywords: Taeniasis; Cysticercosis; Reproduction number; Equilibria; Stability analysis; Sensitivity analysis

\section{Introduction}

Taeniasis and cysticercosis are foodborne infections which are caused by the adult and larval form tapeworms, respectively [29]. Three tapeworm species that cause taeniasis in humans and cysticercosis in humans, pigs and cattle are Taenia solium, Taenia saginata and Taenia asiatica [36]. While T. asiatica is endemic only in Asia, other tapeworm species are distributed worldwide [35]. Taeniasis and cysticercosis are associated with poor hygiene and sanitation, open human defecation, free range farming system for pigs and cattle, and poverty $[11,35]$. They affect mainly poor and marginalized communities who always have

(c) The Author(s) 2021. This article is licensed under a Creative Commons Attribution 4.0 International License, which permits use, sharing, adaptation, distribution and reproduction in any medium or format, as long as you give appropriate credit to the original author(s) and the source, provide a link to the Creative Commons licence, and indicate if changes were made. The images or other third party material in this article are included in the article's Creative Commons licence, unless indicated otherwise in a credit line to the material. If material is not included in the article's Creative Commons licence and your intended use is not permitted by statutory regulation or exceeds the permitted use, you will need to obtain permission directly from the copyright holder. To view a copy of this licence, visit http://creativecommons.org/licenses/by/4.0/. 
close contact with pigs and cattle, and depend on pork and beef as their sources of food $[30,36]$.

Humans acquire taeniasis when they consume raw or undercooked beef or pork that contains the tapeworm larval cysts, which develop into adult tapeworms in the human intestine [36]. When humans who are infected with taeniasis defecate in the fields, the tapeworm eggs that are passed out with human faeces spread over the environment by several means, such as water, winds, animal feet and insects, and contaminate the soil, fodder, pastures and water sources [8]. Domestic cattle acquire cysticercosis when they feed on the contaminated environment while pigs acquire cysticercosis through direct consumption of human faeces or indirectly when they ingest taenia eggs from the contaminated environment [29]. When taenia eggs are consumed by cattle and pigs, they hatch, penetrate the intestinal wall and reach the blood circulation system, where they spread throughout the body tissues and organs such as heart, diaphragm, kidney, lungs, liver and tongue, and develop into cysticerci $[13,35]$. Human cysticercosis is a result of consuming T. solium eggs from the contaminated environment via contaminated water, fruits and vegetables, or by putting contaminated fingers in the mouth [7]. Once the eggs are ingested, they hatch in the small intestine, and develop into larvae which penetrate the intestinal wall and migrate to various parts of the body such as eyes, muscles, skin and the central nervous system through the blood circulatory system, where they form larval cysts [35]. When the cysts reach and infect the brain, they cause neurocysticercosis which is the most severe form of tapeworm infection of the central nervous system and is the major cause of epilepsy worldwide [36].

Taeniasis and cysticercosis are endemic in many developing countries of Latin America, Africa and Asia [29]. The diseases threaten people's health and livelihood of subsistence farming communities, posing considerable challenges in food safety and reducing the market value of pigs and cattle by making pork and beef unsafe to eat [34, 35]. Globally, the diseases affect approximately 50 million people and nearly 50,000 people die annually due to cysticercosis [1]. In 2010, the World Health Organization (WHO) listed cysticercosis as a major neglected tropical disease and later in 2015, it was identified as a leading cause of deaths from foodborne diseases. In Tanzania, the disease has been reported to be a serious health problem whereby the parasite is spread in almost all regions [33]. Porcine cysticercosis has been reported to be highly endemic in southern, central and northern regions of Tanzania with the prevalence rate of $0.3-17.4 \%, 14.9 \%$ and $5.5-16.9 \%$, respectively $[21,30]$. Human infections have also been reported in the country [15, 23]. For example, in 2012 Tanzania had 17,853 cases and 212 deaths due to epilepsy while porcine cysticercosis cases were $18 \mathrm{i}$ and the economic burden for cysticercosis was estimated to be US\$ 7.9 million [30].

The diagnosis of human taeniasis is done by examination of stool samples while human cysticercosis involves doing a biopsy of subcutaneous cysts, immunodiagnosis, radiography, computed tomography (CT) scan and magnetic resonance imaging (MRI). The diagnosis of neurocysticercosis requires both central nervous system imaging with CT brain scans or MRI and serological testing [35]. The diagnosis of cysticercosis in pigs and cattle involves meat inspection, serological tests and tongue inspection for pigs [34]. The treatment of human taeniasis and cysticercosis is through administration of prescribed medication of praziquantel, niclosamide, nitazoxanide or albendazole [35]. The treatment of neurocysticercosis may involve prolonged doses of albendazole and/or praziquantel with 
supporting therapy such as anti-epileptic drugs, corticosteroids, and possibly surgery for some cases [35]. Intervention strategies in pigs and cattle involve using vaccines such as S3Pvac and TSOL18 for pigs and TSA-18 and TSA-9 for cattle $[18,19]$, and anthelmintic treatment with flubendazole, fenbendazole, oxfendazole, praziquantel and nitazoxanide $[34,35]$.

The use of mathematical models plays an important role in studying the transmission dynamics of infectious diseases and is very useful for deciding on the appropriate disease control strategies. Over the past two decades, various mathematical models have been formulated and analyzed to study the dynamics and control of parasitic foodborne diseases such as cholera, brucellosis and echinococcus [6, 20, 24, 28, 32, 37, 38]. In particular, some statistical and deterministic models with some stochastic elements that have been formulated and analyzed to study the transmission dynamics and control of taeniasis and cysticercosis in humans and pigs can be found in Gonzalez et al. [12], Kyvsgaard et al. [16], Braae et al. [2], Winskill et al. [34], José et al. [14] and Sánchez-Torreset al. [27]. The study done by Winskill et al. [34] has assumed that human taeniasis occurs when susceptible humans interact with pigs that are infected with cysticercosis something that is practically wrong. Moreover, previous studies did not include the cattle population to study the transmission dynamics of the taeniasis and cysticercosis. To get new insights on the diseases transmission dynamics, we formulate and analyze the mathematical model for the transmission dynamics of taeniasis and cysticercosis by including cattle population and incorporating compartments of infected pork and beef. The cattle population is included due to its significance in accelerating diseases through feeding on a contaminated environment which later affects human beings. Also, the market value of cattle is reduced and thus affecting the livelihood of subsistence farming communities and leading to food safety problems.

This paper is organised as follows: the deterministic model and analysis are presented in Sect. 2, and numerical analysis in Sect. 3. A summary and a conclusion are presented in Sect. 4.

\section{Model formulation and analysis}

\subsection{Model formulation}

The basic model for the dynamics of taeniasis and cysticercosis is formulated by modifying the work by Winskill et al. [34] that studied intervention strategies against T. solium cysticercosis, by including cattle population and incorporating compartments of infected pork and beef as described by Kyvsgaard et al. [16]. Taeniasis and cysticercosis use humans as the definitive hosts, and pigs and cattle as the intermediate hosts [8].

The model divides human population into $S_{H}, I_{H T}$ and $I_{H C}$ classes that represent susceptible humans, humans infected with taeniasis and humans infected with cysticercosis, respectively. The pig population is divided into $S_{P}$ and $I_{P}$ classes that represent susceptible pigs and pigs that are infected with cysticercosis, respectively, while the cattle population is divided into $S_{C}$ and $I_{C}$, which represent susceptible cattle and cattle that are infected with cysticercosis, respectively. The compartments $P_{I}$ and $B_{I}$ represent infected pork and beef, respectively, and $E_{T}$ represent the number of taenia eggs in the environment.

Susceptible humans are recruited through birth at per capita rate $\psi$ and diminish through consumption of infected raw or insufficiently cooked pork or beef at rates $\alpha_{p}$ and $\alpha_{b}$, respectively. They also diminish by acquiring cysticercosis through consumption 
of T. solium eggs from the contaminated environment at a rate $\theta$ either in contaminated water, fruits, vegetables or by putting contaminated fingers in the mouth [7]. Humans with cysticercosis increase at a rate $\theta$ due to interaction of susceptible humans with $T$. solium eggs in the contaminated environment and they reduce due to disease induced death at rate $\mu_{d}$. Humans with taeniasis increase at rates $\alpha_{P}$ and $\alpha_{b}$ when susceptible humans feed on infected pork and beef, respectively. The parameter $\beta_{T}$ denotes the probability of susceptible humans acquiring taeniasis from an infected pork or beef, that is, the per capita rate at which susceptible individuals acquire infection. All human compartments suffer natural death rate $\mu_{h}$. The number of taenia eggs in the environment grow as a result of open defecation by humans who are infected with taeniasis at a rate $v$ and decrease due to natural death at a rate $\mu_{e}$.

Susceptible pigs are recruited at per capita rate $\Lambda$ due to birth and reduce at a rate $\gamma_{p}$ when they acquire cysticercosis from the contaminated environment. Similarly, susceptible cattle are recruited at per capita rate $\phi$ due to birth and diminish at a rate $\gamma_{c}$ when they acquire cysticercosis from the contaminated environment. Both pigs and cattle are further slaughtered for consumption at rates $\rho$ and $\sigma$, respectively. The infected pigs and cattle increase at rates $\gamma_{p}$ and $\gamma_{c}$, respectively, as a consequence of susceptible pigs and cattle feeding on contaminated environment. All pigs and cattle classes suffer natural death at rates $\mu_{p}$ and $\mu_{c}$, respectively. The infected pork increases when infected pigs are slaughtered at a rate $\omega$ and it decreases when consumed by humans at a rate $\alpha_{p}$ whereas infected beef increases when infected cattle are slaughtered at a rate $\eta$ and decreases when consumed by humans at a rate $\alpha_{b}$. The parameters $\delta$ and $\epsilon$ are the proportions of infected pork and beef unconsumed by susceptible humans.

In model formulation we consider the free range farming system for both pigs and cattle populations, and we do not consider immigration. We assume that humans can be infected by either taeniasis or cysticercosis; the number of taenia eggs consumed by humans, pigs and cattle has negligible effect on the total number of eggs in the environment and that infected humans, pigs and cattle cannot recover naturally without treatment. We further assume that pigs and cattle do not suffer disease induced mortality, that they become carriers for their life and that the rates at which susceptible humans consume infected raw or undercooked pork or beef depend on the amount of infected pork or beef that is present. Humans, pigs and cattle contact rates with taenia eggs in the environment are assumed to be density dependent. The model for the transmission dynamics of taeniasis and cysticercosis in humans, pigs and cattle is summarized by using the flow diagram in Fig. 1.

The state variables and parameters are summarized in Tables 1 and 2, respectively.

Some parameter values in this paper are assumed within realistic ranges due to the fact that only little has been done on this area, the diseases being common in rural areas where there is inadequate or no meat inspection and the treatment is not readily available [22]. The model that describes the transmission dynamics of taeniasis and cysticercosis is governed by the following system of differential equations:

$$
\begin{aligned}
& \frac{d S_{H}}{d t}=\psi-\beta_{T}\left(\alpha_{p} P_{I}+\alpha_{b} B_{I}\right) S_{H}-\theta S_{H} E_{T}-\mu_{h} S_{H}, \\
& \frac{d I_{H T}}{d t}=\beta_{T}\left(\alpha_{p} P_{I}+\alpha_{b} B_{I}\right) S_{H}-\mu_{h} I_{H T}, \\
& \frac{d I_{H C}}{d t}=\theta S_{H} E_{T}-\left(\mu_{h}+\mu_{d}\right) I_{H C},
\end{aligned}
$$




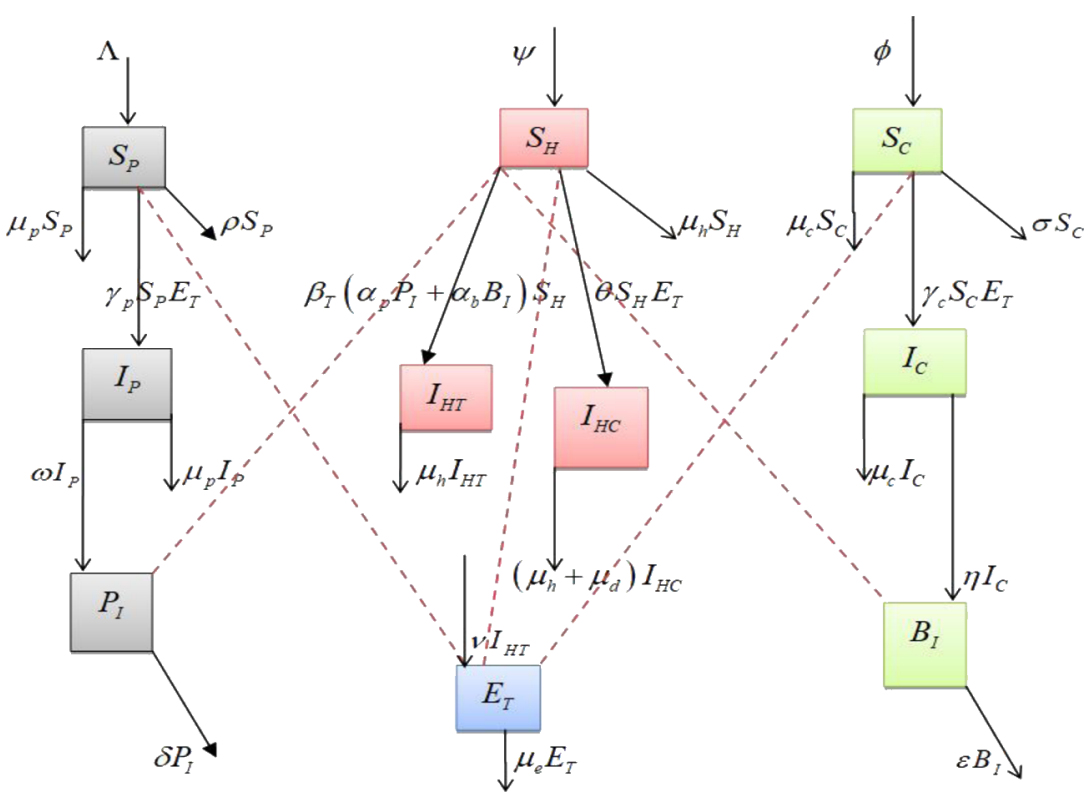

Figure 1 The model flow diagram

Table 1 Description of the state variables

\begin{tabular}{llll}
\hline Variable & Description & Variable & Description \\
\hline$S_{H}$ & Susceptible humans & $P_{l}$ & Cysticercosis infected pork \\
$I_{H T}$ & Humans infected with taeniasis & $S_{C}$ & Susceptible cattle \\
$I_{H C}$ & Humans infected with cysticercosis & $I_{C}$ & Cattle infected with cysticercosis \\
$S_{P}$ & Susceptible pigs & $B_{l}$ & Cysticercosis infected beef \\
$I_{P}$ & Pigs infected with cysticercosis & $E_{T}$ & Taenia eggs in the environment \\
\hline
\end{tabular}

$$
\begin{aligned}
& \frac{d S_{P}}{d t}=\Lambda-\gamma_{p} S_{P} E_{T}-\left(\rho+\mu_{p}\right) S_{P}, \\
& \frac{d I_{P}}{d t}=\gamma_{p} S_{P} E_{T}-\left(\omega+\mu_{p}\right) I_{P}, \\
& \frac{d P_{I}}{d t}=\omega I_{P}-\left(\delta+\alpha_{P}\right) P_{I}, \\
& \frac{d S_{C}}{d t}=\phi-\gamma_{c} S_{C} E_{T}-\left(\sigma+\mu_{c}\right) S_{C}, \\
& \frac{d I_{C}}{d t}=\gamma_{c} S_{C} E_{T}-\left(\eta+\mu_{c}\right) I_{C}, \\
& \frac{d B_{I}}{d t}=\eta I_{C}-\left(\epsilon+\alpha_{b}\right) B_{I}, \\
& \frac{d E_{T}}{d t}=\nu I_{H T}-\mu_{e} E_{T},
\end{aligned}
$$

with initial conditions

$$
\begin{aligned}
& S_{H}(0)>0 ; \quad I_{H T}(0) \geq 0 ; \quad I_{H C}(0) \geq 0 ; \quad S_{P}(0)>0 ; \quad I_{P}(0) \geq 0 ; \\
& P_{I}(0) \geq 0 ; \quad S_{C}(0)>0 ; \quad I_{C}(0) \geq 0 ; \quad B_{I}(0) \geq 0 \quad \text { and } \quad E_{T}(0) \geq 0 \text {. }
\end{aligned}
$$


Table 2 Parameters' description and their values (unit: $y r^{-1}$ )

\begin{tabular}{llll}
\hline Parameter & Description & Value & Source \\
\hline$\psi$ & Per capita recruitment rate of human population & 2247 & {$[37]$} \\
$\mu_{h}$ & Per capita natural death rate of humans & 0.0141 & {$[32]$} \\
$\mu_{d}$ & Human cysticercosis induced death rate & 0.0925 & {$[32]$} \\
$\Lambda$ & Per capita recruitment rate of pig population & 1450 & Assumed \\
$\gamma_{p}$ & T. solium eggs to pig transmission coefficient & 0.01 & {$[16]$} \\
$\alpha_{p}$ & Rate of eating raw/undercooked infected pork & 0.012 & Assumed \\
$\rho$ & Harvesting rate of susceptible pigs & 0.252 & Assumed \\
$\omega$ & Slaughter rate of infected pigs & $0.083 \times 4$ & {$[16]$} \\
$\mu_{p}$ & Per capita natural death rate of pigs & $0.083 \times 12$ & {$[34]$} \\
$\delta$ & Proportion of unconsumed infected pork & 0.358 & Assumed \\
$\phi$ & Per capita recruitment rate of cattle population & 750 & Assumed \\
$\gamma_{c}$ & T. saginata eggs to cattle transmission coefficient & 0.00625 & Assumed \\
$\alpha_{b}$ & Rate of eating raw/undercooked infected beef & 0.023 & Assumed \\
$\sigma$ & Harvesting rate of susceptible cattle & 0.213 & Assumed \\
$\eta$ & Slaughter rate of infected cattle & 0.235 & Assumed \\
$\mu_{c}$ & Per capita natural death rate of cattle & 0.33 & {$[32]$} \\
$\epsilon$ & Proportion of unconsumed infected beef & 0.225 & Assumed \\
$\beta_{T}$ & Probability of humans getting taeniasis & 0.093 & Assumed \\
$\nu$ & Defecation rate by humans with taeniasis & 0.150 & Assumed \\
$\theta$ & T. solium eggs to human cysticercosis transmission coeffient & 0.00523 & Assumed \\
$\mu_{e}$ & Per capita death rate of taenia eggs & 10.42 & {$[32]$} \\
\hline
\end{tabular}

\subsection{Positivity of solutions and invariant region}

For the model system (1) to be biologically and epidemiologically meaningful, we need to show that the model solutions are positive and bounded.

\subsubsection{Positivity of solutions}

From the first equation in the model system (1) for susceptible humans, we have

$$
\begin{aligned}
& \frac{d S_{H}}{d t}=\psi-\beta_{T}\left(\alpha_{p} P_{I}+\alpha_{b} B_{I}\right) S_{H}-\theta S_{H} E_{T}-\mu_{h} S_{H}, \\
& \frac{d S_{H}}{d t} \geq-\left(\beta_{T}\left(\alpha_{p} P_{I}+\alpha_{b} B_{I}\right)+\theta E_{T}+\mu_{h}\right) S_{H}, \\
& \frac{d S_{H}}{S_{H}} \geq-\left(\beta_{T}\left(\alpha_{p} P_{I}+\alpha_{b} B_{I}\right)+\theta E_{T}+\mu_{h}\right) d t, \\
& S_{H}(t) \geq S_{H}(0) e^{\int_{0}^{t}-\left(\beta_{T}\left(\alpha_{p} P_{I}+\alpha_{b} B_{I}\right)+\theta E_{T}+\mu_{h}\right) d s} \geq 0, \quad \forall t \geq 0 .
\end{aligned}
$$

Using the same approach, it can be shown that

$$
\begin{aligned}
& I_{H T}(t) \geq 0 ; \quad I_{H C}(t) \geq 0 ; \quad S_{P}(t) \geq 0 ; \quad I_{P}(t) \geq 0 ; \quad P_{I}(t) \geq 0 ; \\
& S_{C}(t) \geq 0 ; \quad I_{C}(t) \geq 0 ; \quad B_{I}(t) \geq 0 ; \quad E_{T}(t) \geq 0, \quad \forall t \geq 0 .
\end{aligned}
$$

Therefore, all solutions of the model system (1) are positive for all $t \geq 0$.

\subsubsection{Invariant region}

To show that the model solutions are bounded, we let the total populations for humans, pigs and cattle be $H=S_{H}+I_{H T}+I_{H C}, T_{P}=S_{P}+I_{P}$ and $T_{C}=S_{C}+I_{C}$, respectively. By con- 
sidering the human population, we have

$$
\begin{aligned}
& \frac{d H}{d t}=\psi-\mu_{h} H-\mu_{d} I_{H C}, \\
& \frac{d H}{d t}+\mu_{h} H \leq \psi .
\end{aligned}
$$

Integrating throughout, we obtain

$$
H(t) \leq \frac{\psi}{\mu_{h}}+\left(H(0)-\frac{\psi}{\mu_{h}}\right) e^{-\mu_{h} t},
$$

where $H(0)=S_{H}(0)+I_{H T}(0)+I_{H C}(0)$. Following the same procedures, it can be shown that the populations for pigs and cattle are given by

$$
T_{P}(t) \leq \frac{\Lambda}{\mu_{p}}+\left(T_{P}(0)-\frac{\Lambda}{\mu_{p}}\right) e^{-\mu_{p} t} \quad \text { and } \quad T_{C}(t) \leq \frac{\phi}{\mu_{c}}+\left(T_{C}(0)-\frac{\phi}{\mu_{c}}\right) e^{-\mu_{c} t}
$$

respectively, where $T_{P}(0)=S_{P}(0)+I_{P}(0)$ and $T_{C}(0)=S_{C}(0)+I_{C}(0)$. The analysis of the solutions (3) and (4) considers two cases: when $H(0)>\frac{\psi}{\mu_{h}}, T_{P}(0)>\frac{\Lambda}{\mu_{p}}, T_{C}(0)>\frac{\phi}{\mu_{c}}$ and when $H(0)<\frac{\psi}{\mu_{h}}, T_{P}(0)<\frac{\Lambda}{\mu_{p}}, T_{C}(0)<\frac{\phi}{\mu_{c}}$, which gives

$$
\begin{aligned}
& H(t) \leq \Phi_{t}=\max \left\{\frac{\psi}{\mu_{h}}, H(0)\right\}, \\
& T_{P}(t) \leq \Pi_{t}=\max \left\{\frac{\Lambda}{\mu_{p}}, T_{P}(0)\right\}, \\
& T_{C}(t) \leq \Psi_{t}=\max \left\{\frac{\phi}{\mu_{c}}, T_{C}(0)\right\} .
\end{aligned}
$$

Since $H(t)=S_{H}+I_{H T}+I_{H C} \leq \Phi_{t}$, it follows that $I_{H T} \leq \Phi_{t}$. We need to show that $E_{T}$ is also bounded. By considering the last equation in model system (1), we have

$$
\begin{aligned}
& \frac{d E_{T}}{d t}=v I_{H T}-\mu_{e} E_{T}, \\
& \frac{d E_{T}}{d t}+\mu_{e} E_{T}=v I_{H T}, \\
& \frac{d E_{T}}{d t}+\mu_{e} E_{T} \leq \nu \Phi_{t} .
\end{aligned}
$$

Integrating throughout, we obtain

$$
E_{T}(t) \leq \frac{\nu \Phi_{t}}{\mu_{e}}+\left(E_{T}(0)-\frac{\nu \Phi_{t}}{\mu_{e}}\right) e^{-\mu_{e} t}
$$

from which we get

$$
E_{T}(t) \leq \Gamma_{t}=\max \left\{\frac{\nu \Phi_{t}}{\mu_{e}}, E_{T}(0)\right\} .
$$


Using the same approach for the sixth and ninth equations in model system (1), we get

$$
P_{I}(t) \leq \Theta_{t}=\max \left\{\frac{\omega \Pi_{t}}{\left(\alpha_{b}+\delta\right)}, P_{I}(0)\right\} \quad \text { and } \quad B_{I}(t) \leq \xi_{t}=\max \left\{\frac{\eta \Psi_{t}}{\left(\epsilon+\alpha_{b}\right)}, B_{I}(0)\right\} .
$$

Therefore, all solutions of the model system (1) are positive invariant in the region

$$
\begin{aligned}
\Omega= & \left\{\left(S_{H}, I_{H T}, I_{H C}, S_{P}, I_{P}, P_{I}, S_{C}, I_{C}, B_{I}, E_{T}\right) \in \mathbb{R}_{+}^{10}: 0 \leq H(t) \leq \Phi_{t} ; 0 \leq T_{p}(t) \leq \Pi_{t} ;\right. \\
& \left.0 \leq T_{C}(t) \leq \Psi_{t} ; 0 \leq E_{T}(t) \leq \Gamma_{t} ; 0 \leq P_{I}(t) \leq \Theta_{t} ; 0 \leq B_{I}(t) \leq \xi_{t}\right\} .
\end{aligned}
$$

All solutions which start at the boundary of region $\Omega$ converge to this region. The model system (1) is biologically and epidemiologically meaningful and therefore we can consider the flow generated by the model for analysis.

\subsection{Equilibrium states and reproduction number $\boldsymbol{R}_{\mathbf{0}}$}

\subsubsection{The disease free equilibrium $\left(E^{0}\right)$}

When there are no infections in humans, pigs and cattle populations, we obtain the disease free equilibrium $E^{0}$ which is given by

$$
E^{0}=\left(\frac{\psi}{\mu_{h}}, 0,0, \frac{\Lambda}{\left(\rho+\mu_{p}\right)}, 0,0, \frac{\phi}{\left(\sigma+\mu_{c}\right)}, 0,0,0\right) .
$$

\subsubsection{The basic reproduction number $R_{0}$}

The basic reproduction number $R_{0}$ is the expected number of secondary infections that may occur as a result of introducing one infected individual in a fully susceptible population [9]. When $R_{0}<1$, the disease clears whereas, when $R_{0}>1$, the disease persists within the population. In computing $R_{0}$, we adopt the next generation matrix method as used by Van den Driessche and Watmough [31]. Let $\mathcal{F}_{i}$ be the new infections in compartment $i$ and $\mathcal{V}_{i}^{+}$and $\mathcal{V}_{i}^{-}$be the transfer terms in and out of the compartment $i$, respectively, then the infected classes can be written as

$$
\frac{d x_{i}}{d t}=\mathcal{F}_{i}(x)-\mathcal{V}_{i}^{+}(x)-\mathcal{V}_{i}^{-}(x)
$$

Using the next generation matrix method, we define $\mathcal{F}_{i}$ and $\mathcal{V}_{i}$ by

$$
\mathcal{F}_{i}=\left(\begin{array}{c}
\beta_{T}\left(\alpha_{p} P_{I}+\alpha_{b} B_{I}\right) S_{H} \\
\theta S_{H} E_{T} \\
\gamma_{p} S_{P} E_{T} \\
0 \\
\gamma_{c} S_{C} E_{T} \\
0 \\
0
\end{array}\right), \quad \mathcal{V}_{i}=\left(\begin{array}{c}
\mu_{h} I_{H T} \\
\left(\mu_{h}+\mu_{d}\right) I_{H C} \\
\left(\omega+\mu_{p}\right) I_{P} \\
-\omega I_{P}+\left(\delta+\alpha_{p}\right) P_{I} \\
\left(\eta+\mu_{c}\right) I_{C} \\
-\eta I_{C}+\left(\epsilon+\alpha_{b}\right) B_{I} \\
-\nu I_{H T}+\mu_{e} E_{T}
\end{array}\right) .
$$

The Jacobian of matrices $\mathcal{F}_{i}$ and $\mathcal{V}_{i}$ at the disease free equilibrium $E^{0}$ are given by

$$
F=\frac{\partial \mathcal{F}_{i}}{\partial x_{j}}\left(E^{0}\right), \quad V=\frac{\partial \mathcal{V}_{i}}{\partial x_{j}}\left(E^{0}\right) .
$$


The basic reproduction number $R_{0}$ is given by

$$
R_{0}=\rho\left(F V^{-1}\right)
$$

Using the definitions in (9), we have

$$
\begin{aligned}
F & =\left(\begin{array}{ccccccc}
0 & 0 & 0 & \frac{\beta_{T} \alpha_{p} \psi}{\mu_{h}} & 0 & \frac{\beta_{T} \alpha_{b} \psi}{\mu_{h}} & 0 \\
0 & 0 & 0 & 0 & 0 & 0 & \frac{\theta \psi}{\mu_{h}} \\
0 & 0 & 0 & 0 & 0 & 0 & \frac{\gamma_{p} \Lambda}{\left(\rho+\mu_{p}\right)} \\
0 & 0 & 0 & 0 & 0 & 0 & 0 \\
0 & 0 & 0 & 0 & 0 & 0 & \frac{\gamma_{c} \phi}{\left(\sigma+\mu_{c}\right)} \\
0 & 0 & 0 & 0 & 0 & 0 & 0 \\
0 & 0 & 0 & 0 & 0 & 0 & 0
\end{array}\right) \text { and } \\
V & =\left(\begin{array}{cccccccc}
\mu & & & \\
\mu_{h} & 0 & & 0 & 0 & 0 & 0 & 0 \\
0 & \left(\mu_{h}+\mu_{d}\right) & 0 & 0 & 0 & 0 & 0 \\
0 & 0 & \left(\omega+\mu_{p}\right) & 0 & 0 & 0 & 0 \\
0 & 0 & -\omega & \delta+\alpha_{p} & 0 & 0 & 0 \\
0 & 0 & 0 & 0 & \eta+\mu_{c} & 0 & 0 \\
0 & 0 & 0 & 0 & -\eta & \left(\epsilon+\alpha_{b}\right) & 0 \\
-v & 0 & 0 & 0 & 0 & 0 & \mu_{e}
\end{array}\right) .
\end{aligned}
$$

The next generation matrix is given by

$$
F V^{-1}=\left(\begin{array}{ccccccc}
0 & 0 & \frac{\beta_{T} \omega \alpha_{p} S_{H}^{0}}{C_{1}} & \frac{\beta_{T} \alpha_{p} S_{H}^{0}}{\left(\delta+\alpha_{p}\right)} & \frac{\beta_{T} \eta \alpha_{b} S_{H}^{0}}{C_{2}} & \frac{\beta_{T} \alpha_{b} S_{H}^{0}}{\left(\epsilon+\alpha_{b}\right)} & 0 \\
\frac{\nu \theta S_{H}^{0}}{\mu_{e} \mu_{h}} & 0 & 0 & 0 & 0 & 0 & \frac{\theta S_{H}^{0}}{\mu_{e}} \\
\frac{\nu \gamma_{p} S_{P}^{0}}{\mu_{e} \mu_{h}} & 0 & 0 & 0 & 0 & 0 & \frac{\gamma_{p} S_{P}^{0}}{\mu_{e}} \\
0 & 0 & 0 & 0 & 0 & 0 & 0 \\
\frac{\nu \gamma_{c} S_{C}^{0}}{\mu_{e} \mu_{h}} & 0 & 0 & 0 & 0 & 0 & \frac{\gamma_{c} S_{C}^{0}}{\mu_{e}} \\
0 & 0 & 0 & 0 & 0 & 0 & 0 \\
0 & 0 & 0 & 0 & 0 & 0 & 0
\end{array}\right),
$$

where $C_{1}=\left(\omega+\mu_{p}\right)\left(\delta+\alpha_{p}\right), C_{2}=\left(\eta+\mu_{c}\right)\left(\epsilon+\alpha_{b}\right), S_{H}^{0}=\frac{\psi}{\mu_{h}}, S_{P}^{0}=\frac{\Lambda}{\left(\rho+\mu_{p}\right)}$ and $S_{C}^{0}=\frac{\phi}{\left(\sigma+\mu_{c}\right)}$. Using the definition in (10), the basic reproduction number $R_{0}$ is given by

$$
R_{0}=\sqrt{R_{H P}+R_{H C}}
$$

where

$$
\begin{aligned}
& R_{H P}=\frac{\beta_{T} v \alpha_{p} \gamma_{p} \omega \psi \Lambda}{\mu_{h}^{2} \mu_{e}\left(\omega+\mu_{p}\right)\left(\alpha_{p}+\delta\right)\left(\mu_{p}+\rho\right)} \quad \text { and } \\
& R_{H C}=\frac{\beta_{T} v \alpha_{b} \gamma_{c} \eta \psi \phi}{\mu_{h}^{2} \mu_{e}\left(\eta+\mu_{c}\right)\left(\alpha_{b}+\epsilon\right)\left(\mu_{c}+\sigma\right)} .
\end{aligned}
$$

$R_{H P}$ is the partial reproduction number due to interaction of humans and pigs whereas $R_{H C}$ is the partial reproduction number due to interaction of humans and cattle. To give 
the biological meaning for $R_{0}$, we rewrite $R_{H P}$ and $R_{H C}$ in the forms

$$
\begin{aligned}
& R_{H P}=\beta_{T} \gamma_{p} \frac{\psi}{\mu_{h}} \frac{v}{\mu_{e}} \frac{1}{\mu_{h}} \frac{\Lambda}{\left(\mu_{p}+\rho\right)} \frac{\omega}{\left(\omega+\mu_{p}\right)} \frac{\alpha_{p}}{\left(\alpha_{p}+\delta\right)}, \\
& R_{H C}=\beta_{T} \gamma_{c} \frac{\psi}{\mu_{h}} \frac{v}{\mu_{e}} \frac{1}{\mu_{h}} \frac{\phi}{\left(\mu_{c}+\sigma\right)} \frac{\eta}{\left(\eta+\mu_{c}\right)} \frac{\alpha_{b}}{\left(\alpha_{b}+\epsilon\right)} .
\end{aligned}
$$

The terms in (12) can be interpreted as follows: $\nu / \mu_{e}$ is the density of taenia eggs released by humans with taeniasis, $1 / \mu_{h}$ is the human life expectancy and $\beta_{T}$ is the probability of humans to be infected with taeniasis due to consumption of raw or insufficiently cooked pork or beef infected with tapeworm larval cysts. The terms $\psi / \mu_{h}, \Lambda /\left(\mu_{p}+\rho\right)$ and $\phi /\left(\mu_{c}+\sigma\right)$ are initial populations for susceptible humans, susceptible pigs and susceptible cattle, respectively; $1 /\left(\mu_{p}+\rho\right)$ and $1 /\left(\mu_{c}+\sigma\right)$ are the average times pigs and cattle spend in susceptible classes, respectively; $1 /\left(\omega+\mu_{p}\right)$ and $1 /\left(\eta+\mu_{c}\right)$ are the infectious periods of infected pigs and cattle, respectively; $1 /\left(\alpha_{p}+\delta\right)$ and $1 /\left(\alpha_{b}+\epsilon\right)$ are the average infectious period for infected pork and beef, respectively, whereas $\omega /\left(\omega+\mu_{p}\right)$ and $\eta /\left(\eta+\mu_{c}\right)$ are the proportions of infected pigs and cattle that are slaughtered for consumption, respectively. The terms $\alpha_{p} /\left(\alpha_{p}+\delta\right)$ and $\alpha_{b} /\left(\alpha_{b}+\epsilon\right)$ are the proportions of infected pork and beef that are eaten by susceptible humans, respectively, whereas $\gamma_{p}$ and $\gamma_{c}$ are the rates at which $T$. solium eggs and T. sagnata eggs are consumed by pigs and cattle, respectively, from the contaminated environment.

\subsubsection{Sensitivity analysis}

To determine how sensitive the model parameters are to the diseases' transmission, we adopt the normalized forward sensitivity index approach as used by Chitnis et al. [5]. If $\kappa$ is a parameter in the basic reproduction number $R_{0}$, then the sensitivity index of $R_{0}$ with respect to $\kappa$ is given by

$$
\Gamma_{\kappa}^{R_{0}}=\frac{\partial R_{0}}{\partial \kappa} \times \frac{\kappa}{R_{0}} .
$$

Using Eq. (13) and parameter values in Table 2, we obtain sensitivity indices for each parameter as shown in Table 3. The positive sign of sensitivity index indicates that an increase or decrease of parameter value while keeping other parameters constant increases or decreases the basic reproduction number $R_{0}$. The negative sign indicates that an increase or decrease of parameter value causes a decrease or increase in expected new average infection $R_{0}$. For instance, $\gamma_{p}=+0.1421$ means that an increase in $\gamma_{p}$ by $10 \%$, increases $R_{0}$ by $1.421 \%$ and hence the disease transmission; and $\delta=-0.1375$ means that an increase in $\delta$ by $20 \%$ causes a decrease in $R_{0}$ by $2.75 \%$ and thus decrease the disease transmission.

The most positive sensitive parameters in the model are human's recruitment $(\psi)$, the probability of humans infection with taeniasis $\left(\beta_{T}\right)$ and the defecation rate by humans who are infected with taeniasis $(v)$ whereas the most negative sensitive parameter is the natural mortality rate of humans $\left(\mu_{h}\right)$. However, since it is unethical and not practical to increase human natural mortality, other parameters with negative sensitivity indices such as death rate of taenia eggs and proportions of unconsumed infected beef and pork can be considered for disease control.

The sensitivity indices for all parameters in the basic reproduction number $R_{0}$ are plotted on a bar graph in Fig. 2. 
Table 3 Sensitivity indices

\begin{tabular}{lclc}
\hline Parameter & Sensitivity Index & Parameter & Sensitivity Index \\
\hline$\Lambda$ & +0.1421 & $\omega$ & +0.1066 \\
$\psi$ & +0.3579 & $\eta$ & +0.2090 \\
$\phi$ & +0.5000 & $\nu$ & +0.5000 \\
$\gamma_{p}$ & +0.1421 & $\delta$ & -0.1375 \\
$\gamma_{c}$ & $\epsilon$ & -0.3343 \\
$\alpha_{p}$ & +0.3579 & $\mu_{p}$ & -0.2200 \\
$\alpha_{b}$ & +0.1375 & $\mu_{h}$ & -1.0000 \\
$\beta_{T}$ & +0.3343 & $\mu_{c}$ & -0.4266 \\
$\sigma$ & +0.5000 & $\mu_{e}$ & -0.5000 \\
$\rho$ & -0.1404 & & \\
\hline
\end{tabular}

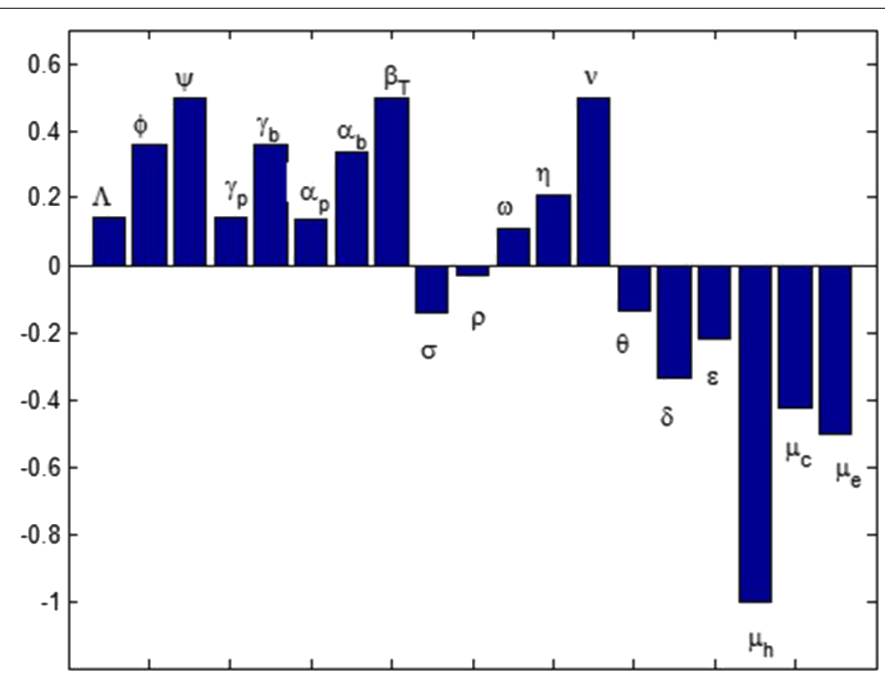

Figure 2 Graphical representation of sensitivity indices

\subsubsection{The endemic equilibrium $\left(E^{*}\right)$}

The endemic equilibrium is a point at which taeniasis and cysticercosis exist in humans, pigs and cattle populations. It is obtained by setting the derivatives in model system (1) equal to zero and solving for state variables. The endemic equilibrium $E^{*}=$ $\left(S_{H}^{*}, I_{H T}^{*}, I_{H C}^{*}, S_{P}^{*}, I_{P}^{*}, P_{I}^{*}, S_{C}^{*}, I_{C}^{*}, B_{I}^{*}, E_{T}^{*}\right)$ is given by

$$
\begin{aligned}
& S_{H}^{*}=\frac{\psi}{\left(\beta_{T} F_{0} E_{T}^{*}+\theta E_{T}^{*}+\mu_{h}\right)}, \quad I_{H T}^{*}=\frac{\beta_{T} F_{0} E_{T}^{*} S_{H}^{*}}{\mu_{h}}, \quad I_{H C}^{*}=\frac{\theta S_{H}^{*} E_{T}^{*}}{\left(\mu_{h}+\mu_{d}\right)}, \\
& S_{P}^{*}=\frac{\Lambda}{\left(\gamma_{p} E_{T}^{*}+\rho+\mu_{p}\right)}, \quad I_{P}^{*}=\frac{\gamma_{p} \Lambda E_{T}^{*}}{\left(\gamma_{p} E_{T}^{*}+\rho+\mu_{p}\right)\left(\omega+\mu_{p}\right)}, \\
& P_{I}^{*}=\frac{\omega \gamma_{p} \Lambda E_{T}^{*}}{\left(\gamma_{p} E_{T}^{*}+\rho+\mu_{p}\right)\left(\omega+\mu_{p}\right)\left(\delta+\alpha_{p}\right)}, \quad S_{C}^{*}=\frac{\phi}{\left(\gamma_{c} E_{T}^{*}+\sigma+\mu_{c}\right)}, \\
& I_{C}^{*}=\frac{\gamma_{c} \phi E_{T}^{*}}{\left(\gamma_{c} E_{T}^{*}+\sigma+\mu_{c}\right)\left(\eta+\mu_{c}\right)}, \quad B_{I}^{*}=\frac{\eta \gamma_{c} \phi E_{T}^{*}}{\left(\gamma_{c} E_{T}^{*}+\sigma+\mu_{c}\right)\left(\eta+\mu_{c}\right)\left(\epsilon+\alpha_{b}\right)},
\end{aligned}
$$

where

$$
F_{0}=\frac{\alpha_{p} \omega \gamma_{p} \Lambda}{\left(\gamma_{p} E_{T}^{*}+\rho+\mu_{p}\right)\left(\omega+\mu_{p}\right)\left(\alpha_{p}+\delta\right)}+\frac{\alpha_{b} \eta \gamma_{c} \phi}{\left(\gamma_{c} E_{T}^{*}+\sigma+\mu_{c}\right)\left(\eta+\mu_{c}\right)\left(\alpha_{b}+\epsilon\right)} .
$$


Table 4 Number of possible positive real roots when $R_{0}<1$ and $R_{0}>1$

\begin{tabular}{llllllll}
\hline Cases & $a_{0}$ & $a_{1}$ & $a_{2}$ & $a_{3}$ & $R_{0}$ & No. of Sign Change & No. of + ve Real Roots \\
\hline 1 & + & + & + & + & $R_{0}<1$ & 0 & 0 \\
2 & + & + & + & - & $R_{0}>1$ & 1 & 1 \\
3 & + & + & - & + & $R_{0}<1$ & 2 & 0,2 \\
4 & + & + & - & - & $R_{0}>1$ & 1 & 1 \\
\hline
\end{tabular}

To obtain $E_{T}^{*}$, we solve for the real roots of the polynomial:

$$
a_{0} E_{T}^{* 3}+a_{1} E_{T}^{* 2}+a_{2} E_{T}^{*}+a_{3}=0
$$

where

$$
\begin{aligned}
a_{0}= & 1>0, \\
a_{1}= & \frac{\alpha_{p} \omega \Lambda \beta_{T}}{\theta\left(\mu_{p}+\omega\right)\left(\alpha_{p}+\delta\right)}+\frac{\alpha_{b} \eta \phi \beta_{T}}{\theta\left(\mu_{c}+\eta\right)\left(\alpha_{p}+\epsilon\right)}+\frac{\left(\mu_{c}+\sigma\right)}{\gamma_{c}}+\frac{\left(\mu_{p}+\rho\right)}{\gamma_{p}}+\frac{\mu_{h}}{\theta}>0, \\
a_{2}= & \frac{\left(\rho+\mu_{p}\right)\left(\sigma+\mu_{c}\right)}{\gamma_{c} \gamma_{p}}+\frac{\mu_{h}\left(\sigma+\mu_{c}\right)}{\theta \gamma_{c}}+\frac{\mu_{h}\left(\rho+\mu_{p}\right)}{\theta \gamma_{p}}+\frac{\alpha_{p} \omega \Lambda \beta_{T}\left(\sigma+\mu_{c}\right)}{\theta \gamma_{c}\left(\mu_{p}+\omega\right)\left(\alpha_{p}+\delta\right)} \\
& +\frac{\alpha_{b} \eta \phi \beta_{T}\left(\rho+\mu_{p}\right)}{\theta \gamma_{p}\left(\mu_{c}+\eta\right)\left(\alpha_{p}+\epsilon\right)}-\left(\frac{\nu \psi \beta_{T} \alpha_{p} \omega \Lambda \gamma_{c}}{\mu_{h} \mu_{e} \gamma_{c} \theta\left(\mu_{p}+\omega\right)\left(\alpha_{p}+\delta\right)}+\frac{\nu \psi \beta_{T} \alpha_{b} \eta \phi \gamma_{p}}{\mu_{h} \mu_{e} \gamma_{p} \theta\left(\mu_{c}+\eta\right)\left(\alpha_{p}+\epsilon\right)}\right), \\
a_{3}= & \frac{\mu_{h}\left(\sigma+\mu_{c}\right)\left(\rho+\mu_{p}\right)\left(1+R_{0}\right)\left(1-R_{0}\right)}{\theta \gamma_{c} \gamma_{p}} .
\end{aligned}
$$

To analyze the possible number of positive real roots of polynomial (14) when $R_{0}<1$ and $R_{0}>1$, we adopt the approach in Okosun et al. [25]. Using this approach, the number of possible real roots when $R_{0}<1$ and $R_{0}>1$ are summarized in Table 4 .

Therefore the model system (1) has a unique endemic equilibrium when $R_{0}>1$ as shown in cases 2 and 4 . Hence we state the following theorem.

Theorem 1 The model system (1) has a unique endemic equilibrium when the basic reproduction number $R_{0}>1$.

\subsection{Stability analysis of equilibrium states}

2.4.1 The global stability of the disease free equilibrium $\left(E^{0}\right)$

Theorem 2 The disease free equilibrium $\left(E^{0}\right)$ of the model system (1) is globally asymptotically stable when $R_{0}<1$.

Proof To analyze the global stability of the disease free equilibrium, we adopt the approach used in Castillo-Chavez et al. [4] and Dumont et al. [10]. Using this method, the system of differential equations (1) is written as

$$
\begin{aligned}
& \frac{d X_{r}}{d t}=B\left(X_{r}-X_{\mathrm{DFE}}\right)+B_{1} X_{n}, \\
& \frac{d X_{n}}{d t}=B_{2} X_{n},
\end{aligned}
$$

where $X_{r}$ and $X_{n}$ are the non-transmitting and transmitting classes, respectively, $X_{\mathrm{DFE}}$ is the disease free equilibrium, whereas $B, B_{1}$ and $B_{2}$ are the matrices to be computed. Here, 
we have

$$
\begin{aligned}
& X_{r}=\left(S_{H}, S_{P}, S_{C}\right)^{T}, \\
& X_{\mathrm{DFE}}=\left(\frac{\psi}{\mu_{h}}, \frac{\Lambda}{\mu_{p}+\rho}, \frac{\phi}{\mu_{c}+\sigma}\right)^{T} .
\end{aligned}
$$

Thus,

$$
X_{r}-X_{\mathrm{DFE}}=\left(S_{H}-\frac{\psi}{\mu_{h}}, S_{P}-\frac{\Lambda}{\mu_{p}+\rho}, S_{C}-\frac{\phi}{\mu_{c}+\sigma}\right)^{T} .
$$

Therefore from Eq. (15), we have

$$
\left(\begin{array}{c}
\psi-\beta_{T}\left(\alpha_{p} P_{I}+\alpha_{b} B_{I}\right) S_{H}-\theta S_{H} E_{T}-\mu_{h} S_{H} \\
\Lambda-\gamma_{p} S_{P} E_{T}-\left(\rho+\mu_{p}\right) S_{P} \\
\phi-\gamma_{c} S_{C} E_{T}-\left(\sigma+\mu_{c}\right) S_{P}
\end{array}\right)=B\left(\begin{array}{c}
S_{H}-\frac{\psi}{\mu_{h}} \\
S_{P}-\frac{\Lambda}{\mu_{p}+\rho} \\
S_{C}-\frac{\phi}{\mu_{c}+\sigma}
\end{array}\right)+B_{1}\left(\begin{array}{c}
I_{H T} \\
I_{H C} \\
I_{P} \\
P_{I} \\
I_{C} \\
B_{I} \\
E_{T}
\end{array}\right)
$$

and

$$
\left(\begin{array}{c}
\beta_{T}\left(\alpha_{p} P_{I}+\alpha_{b} B_{I}\right) S_{H}-\mu_{h} I_{H T} \\
\theta S_{H} E_{T}-\left(\mu_{h}+\mu_{d}\right) I_{H C} \\
\gamma_{p} S_{P} E_{T}-\left(\omega+\mu_{p}\right) I_{P} \\
\omega I_{P}-\left(\delta+\alpha_{p}\right) P_{I} \\
\gamma_{c} S_{C} E_{T}-\left(\eta+\mu_{c}\right) I_{C} \\
\eta I_{C}-\left(\epsilon+\alpha_{b}\right) B_{I} \\
v I_{H T}-\mu_{e} E_{T}
\end{array}\right)=B_{2}\left(\begin{array}{c}
I_{H T} \\
I_{H C} \\
I_{P} \\
P_{I} \\
I_{C} \\
B_{I} \\
E_{T}
\end{array}\right) .
$$

From (18) and (19), the matrices $B, B_{1}$ and $B_{2}$ are given by

$$
\begin{aligned}
B & =\left(\begin{array}{ccc}
-\mu_{h} & 0 & 0 \\
0 & -\left(\rho+\mu_{p}\right) & 0 \\
0 & 0 & -\left(\sigma+\mu_{c}\right)
\end{array}\right), \\
B_{1} & =\left(\begin{array}{lllcccc}
0 & 0 & 0 & -\beta_{T} \alpha_{p} S_{H} & 0 & -\beta_{T} \alpha_{b} S_{H} & -\theta S_{H} \\
0 & 0 & 0 & 0 & 0 & 0 & \gamma_{p} S_{P} \\
0 & 0 & 0 & 0 & 0 & 0 & \gamma_{c} S_{C}
\end{array}\right),
\end{aligned}
$$


and

$$
B_{2}=\left(\begin{array}{ccccccc}
-\mu_{h} & 0 & 0 & \beta_{T} \alpha_{p} S_{H} & 0 & \beta_{T} \alpha_{b} S_{H} & 0 \\
0 & -\left(\mu_{h}+\mu_{d}\right) & 0 & 0 & 0 & 0 & \theta S_{H} \\
0 & 0 & -\left(\omega+\mu_{p}\right) & 0 & 0 & 0 & \gamma_{p} S_{P} \\
0 & 0 & \omega & -\left(\delta+\alpha_{p}\right) & 0 & 0 & 0 \\
0 & 0 & 0 & 0 & -\left(\eta+\mu_{c}\right) & 0 & \gamma_{c} S_{C} \\
0 & 0 & 0 & 0 & \eta & -\left(\epsilon+\alpha_{b}\right) & 0 \\
\nu & 0 & 0 & 0 & 0 & 0 & -\mu_{e}
\end{array}\right) .
$$

It can be observed that matrix $B$ has real and negative eigenvalues. Thus, the system

$$
\frac{d X_{r}}{d t}=B\left(X_{r}-X_{\mathrm{DFE}}\right)+B_{1} X_{n}
$$

is globally asymptotically stable at $X_{\mathrm{DFE}}$.

To prove the stability of $B_{2}$, we adopt the idea of stable Metzler matrix and apply the lemma in Dumont et al. [10]. A Metzler matrix is a matrix whose of-diagonal elements are non-negative, denoted by $B_{2}(i, j) \geq 0$, for all $i \neq j$. Thus, it can be observed that $B_{2}$ is a Metzler matrix.

Lemma 1 Let $M$ be a square Metzler matrix written in block form:

$$
M=\left(\begin{array}{ll}
P & Q \\
R & S
\end{array}\right),
$$

where $P$ and $S$ are square matrices. $M$ is Metzler stable if and only if matrices $P$ and $S-$ $R P^{-1} Q$ are Metzler stable.

Proof Comparing the Metzler matrix $B_{2}$ with a square Metzler matrix $M$, the matrices $P$, $Q, R$ and $S$ are defined as

$$
\begin{aligned}
P & =\left(\begin{array}{ccc}
-\mu_{h} & 0 & 0 \\
0 & -\left(\mu_{h}+\mu_{d}\right) & 0 \\
0 & 0 & -\left(\omega+\mu_{p}\right)
\end{array}\right), \quad Q=\left(\begin{array}{cccc}
\beta_{T} \alpha_{p} S_{H} & 0 & \beta_{T} \alpha_{b} S_{H} & 0 \\
0 & 0 & 0 & \theta S_{H} \\
0 & 0 & 0 & \gamma_{p} S_{P}
\end{array}\right), \\
R & =\left(\begin{array}{lll}
0 & 0 & \omega \\
0 & 0 & 0 \\
0 & 0 & 0 \\
\nu & 0 & 0
\end{array}\right), \quad S=\left(\begin{array}{cccc}
-\left(\delta+\alpha_{p}\right) & 0 & 0 & 0 \\
0 & -\left(\eta+\mu_{c}\right) & 0 & \gamma_{c} S_{C} \\
0 & \eta & -\left(\epsilon+\alpha_{b}\right) & 0 \\
0 & 0 & 0 & -\mu_{e}
\end{array}\right) .
\end{aligned}
$$

Clearly, $P$ is a stable Metzler matrix. After some computations, we obtain

$$
S-R P^{-1} Q=\left(\begin{array}{cccc}
-\left(\delta+\alpha_{p}\right) & 0 & 0 & \frac{\omega \gamma_{p} S_{P}}{\left(\omega+\mu_{p}\right)} \\
0 & -\left(\eta+\mu_{c}\right) & 0 & \gamma_{c} S_{C} \\
0 & \eta & -\left(\epsilon+\alpha_{b}\right) & 0 \\
\frac{\nu \beta_{T} \alpha_{p} S_{H}}{\mu_{h}} & 0 & \frac{\nu \beta_{T} \alpha_{b} S_{H}}{\mu_{h}} & -\mu_{e}
\end{array}\right) \text {. }
$$


Thus, $S-R P^{-1} Q$ is Metzler stable if $\operatorname{Det}\left(S-R P^{-1} Q\right)>0$. That is,

$$
\mathcal{W}\left(\delta+\alpha_{p}\right) \mu_{e}-\frac{\nu \beta_{T} S_{H}\left(\omega \alpha_{p} \gamma_{p} \mathcal{W} S_{P}+\eta \alpha_{b} \gamma_{c}\left(\delta+\alpha_{b}\right)\left(\omega+\mu_{p}\right) S_{C}\right)}{\mu_{h}\left(\omega+\mu_{p}\right)}>0,
$$

where $\mathcal{W}=\left(\epsilon+\alpha_{c}\right)\left(\eta+\mu_{c}\right)$. Substituting the values for $S_{H}, S_{P}$ and $S_{C}$ at disease free equilibrium into (21) and simplifying the expression, we obtain $1-R_{0}^{2}>0$, where $R_{0}$ is given in (11). Therefore, the disease free equilibrium $E^{0}$ is globally asymptotically stable when $R_{0}<1$.

\subsubsection{Global stability of the endemic equilibrium $\left(E^{*}\right)$}

Theorem 3 The endemic equilibrium ( $\left.E^{*}\right)$ for the model system (1) is globally asymptotically stable when $R_{0}>1$.

Proof Since the endemic equilibrium exists if and only if $R_{0}>1$, we adopt the approach in Osman et al. [26] to prove global stability of endemic equilibrium $E^{*}$. Consider the nonlinear Lyapunov function

$$
\begin{aligned}
\mathcal{L}= & S_{H}^{*}\left(\frac{S_{H}}{S_{H}^{*}}-\ln \frac{S_{H}}{S_{H}^{*}}\right)+I_{H T}^{*}\left(\frac{I_{H T}}{I_{H T}^{*}}-\ln \frac{I_{H T}}{I_{H T}^{*}}\right)+I_{H C}^{*}\left(\frac{I_{H C}}{I_{H C}^{*}}-\ln \frac{I_{H C}}{I_{H C}^{*}}\right) \\
& +S_{P}^{*}\left(\frac{S_{P}}{S_{P}^{*}}-\ln \frac{S_{P}}{S_{P}^{*}}\right)+I_{P}^{*}\left(\frac{I_{P}}{I_{P}^{*}}-\ln \frac{I_{P}}{I_{P}^{*}}\right)+P_{I}^{*}\left(\frac{P_{I}}{P_{I}^{*}}-\ln \frac{P_{I}}{P_{I}^{*}}\right)+S_{C}^{*}\left(\frac{S_{C}}{S_{C}^{*}}-\ln \frac{S_{C}}{S_{C}^{*}}\right) \\
& +I_{C}^{*}\left(\frac{I_{C}}{I_{C}^{*}}-\ln \frac{I_{C}}{I_{C}^{*}}\right)+B_{I}^{*}\left(\frac{B_{I}}{B_{I}^{*}}-\ln \frac{B_{I}}{B_{I}^{*}}\right)+E_{T}^{*}\left(\frac{E_{T}}{E_{T}^{*}}-\ln \frac{E_{T}}{E_{T}^{*}}\right),
\end{aligned}
$$

The time derivative of the Lyapunov function $\mathcal{L}$ is

$$
\begin{aligned}
\frac{d \mathcal{L}}{d t}= & \left(1-\frac{S_{H}^{*}}{S_{H}}\right) \frac{d S_{H}}{d t}+\left(1-\frac{I_{H T}^{*}}{I_{H T}}\right) \frac{d I_{H T}}{d t}+\left(1-\frac{I_{H C}^{*}}{I_{H C}}\right) \frac{d I_{H C}}{d t}+\left(1-\frac{S_{P}^{*}}{S_{P}}\right) \frac{d S_{P}}{d t} \\
& +\left(1-\frac{I_{P}^{*}}{I_{P}}\right) \frac{d I_{P}}{d t}+\left(1-\frac{P_{I}^{*}}{P_{I}}\right) \frac{d P_{I}}{d t}+\left(1-\frac{S_{C}^{*}}{S_{C}}\right) \frac{d S_{C}}{d t}+\left(1-\frac{I_{C}^{*}}{I_{C}}\right) \frac{d I_{C}}{d t} \\
& +\left(1-\frac{B_{I}^{*}}{B_{I}}\right) \frac{d B_{I}}{d t}+\left(1-\frac{E_{T}^{*}}{E_{T}}\right) \frac{d E_{T}}{d t} .
\end{aligned}
$$

Substituting the equations of model system (1) into Eq. (23), we have

$$
\begin{aligned}
\frac{d \mathcal{L}}{d t}= & \left(1-\frac{S_{H}^{*}}{S_{H}}\right)\left(\psi-\beta_{T} \alpha_{p} P_{I} S_{H}-\beta_{T} \alpha_{b} B_{I} S_{H}-\theta S_{H} E_{T}-\mu_{h} S_{H}\right) \\
& +\left(1-\frac{I_{H T}^{*}}{I_{H T}}\right)\left(\beta_{T}\left(\alpha_{p} P_{I}+\alpha_{b} B_{I}\right) S_{H}-\mu_{h} I_{H T}\right) \\
& +\left(1-\frac{I_{H C}^{*}}{I_{H C}}\right)\left(\theta S_{H} E_{T}-\left(\mu_{h}+\mu_{d}\right) I_{H C}\right) \\
& +\left(1-\frac{S_{P}^{*}}{S_{P}}\right)\left(\Lambda-\gamma_{p} S_{P} E_{T}-\left(\rho+\mu_{p}\right) S_{P}\right)+\left(1-\frac{I_{P}^{*}}{I_{P}}\right)\left(\gamma_{p} S_{P} E_{T}-\left(\omega+\mu_{p}\right) I_{P}\right) \\
& +\left(1-\frac{P_{I}^{*}}{P_{I}}\right)\left(\omega I_{P}-\left(\delta+\alpha_{p}\right) P_{I}\right)+\left(1-\frac{S_{C}^{*}}{S_{C}}\right)\left(\phi-\gamma_{c} S_{C} E_{T}-\left(\sigma+\mu_{c}\right) S_{C}\right) \\
& +\left(1-\frac{I_{C}^{*}}{I_{C}}\right)\left(\gamma_{c} S_{C} E_{T}-\left(\eta+\mu_{c}\right) I_{C}\right)+\left(1-\frac{B_{I}^{*}}{B_{I}}\right)\left(\eta I_{C}-\left(\epsilon+\alpha_{b}\right) B_{I}\right)
\end{aligned}
$$




$$
+\left(1-\frac{E_{T}^{*}}{E_{T}}\right)\left(\nu I_{H T}-\mu_{e} E_{T}\right)
$$

Equation (24) can also be written as

$$
\begin{aligned}
\frac{d \mathcal{L}}{d t}= & \psi-\beta_{T} \alpha_{p} P_{I} S_{H}-\beta_{T} \alpha_{b} B_{I} S_{H}-\theta S_{H} E_{T}-\mu_{h} S_{H} \\
& -\frac{\psi S_{H}^{*}}{S_{H}}+\beta_{T} \alpha_{p} P_{I} S_{H}^{*}+\beta_{T} \alpha_{b} B_{I} S_{H}^{*}+\theta E_{T} S_{H}^{*}+\mu_{h} S_{H}^{*}+\beta_{T} \alpha_{p} P_{I} S_{H} \\
& +\beta_{T} \alpha_{b} B_{I} S_{H}-\mu_{h} I_{H T}-\frac{\beta_{T} \alpha_{p} P_{I} S_{H} I_{H T}^{*}}{I_{H T}}-\frac{\beta_{T} \alpha_{b} B_{I} S_{H} I_{H T}^{*}}{I_{H T}}+\mu_{h} I_{H T}^{*}+\theta S_{H} E_{T} \\
& -\left(\mu_{h}+\mu_{d}\right) I_{H C}-\frac{\theta S_{H} E_{T} I_{H C}^{*}}{I_{H C}}+\left(\mu_{h}+\mu_{d}\right) I_{H C}^{*}+\Lambda-\gamma_{p} S_{P} E_{T}-\left(\rho+\mu_{p}\right) S_{P} \\
& -\frac{\Lambda S_{P}^{*}}{S_{P}}+\gamma_{p} E_{T} S_{P}^{*}+\left(\rho+\mu_{p}\right) S_{P}^{*}+\gamma_{p} S_{P} E_{T}-\left(\omega+\mu_{p}\right) I_{P}-\frac{\gamma_{p} S_{P} E_{T} I_{P}^{*}}{I_{P}} \\
& +\left(\omega+\mu_{p}\right) I_{P}^{*}+\omega I_{P}-\left(\delta+\alpha_{p}\right) P_{I}-\frac{\omega I_{P} P_{I}^{*}}{P_{I}}+\left(\delta+\alpha_{p}\right) P_{I}^{*}+\phi-\gamma_{c} S_{C} E_{T} \\
& -\left(\sigma+\mu_{c}\right) S_{C}-\frac{\phi S_{C}^{*}}{S_{C}}+\gamma_{c} E_{T} S_{C}^{*}+\left(\sigma+\mu_{c}\right) S_{C}^{*}+\gamma_{c} S_{C} E_{T}-\left(\eta+\mu_{c}\right) I_{C} \\
& -\frac{\gamma_{c} S_{C} E_{T} I_{C}^{*}}{I_{C}}+\left(\eta+\mu_{c}\right) I_{C}^{*}+\eta I_{C}-\left(\epsilon+\alpha_{b}\right) B_{I}-\frac{\eta I_{C} B_{I}^{*}}{B_{I}}+\left(\epsilon+\alpha_{b}\right) B_{I}^{*}+\nu I_{H T} \\
& -\mu_{e} E_{T}-\frac{\nu I_{H T} E_{T}^{*}}{E_{T}}+\mu_{e} E_{T}^{*} .
\end{aligned}
$$

Similarly, Eq. (25) can be written as

$$
\frac{d \mathcal{L}}{d t}=\mathcal{J}-\mathcal{P}
$$

where

$$
\begin{aligned}
\mathcal{J}= & \psi+\beta_{T} \alpha_{p} P_{I} S_{H}^{*}+\beta_{T} \alpha_{b} B_{I} S_{H}^{*}+\theta E_{T} S_{H}^{*}+\mu_{h} S_{H}^{*}+\beta_{T} \alpha_{p} P_{I} S_{H}+\beta_{T} \alpha_{b} B_{I} S_{H} \\
& +\mu_{h} I_{H T}^{*}+\theta S_{H} E_{T}+\left(\mu_{h}+\mu_{d}\right) I_{H C}^{*}+\Lambda+\gamma_{p} E_{T} S_{P}^{*}+\left(\rho+\mu_{p}\right) S_{P}^{*}+\gamma_{p} S_{P} E_{T} \\
& +\left(\omega+\mu_{p}\right) I_{P}^{*}+\omega I_{P}+\left(\delta+\alpha_{p}\right) P_{I}^{*}+\phi+\gamma_{c} E_{T} S_{C}^{*}+\left(\sigma+\mu_{c}\right) S_{C}^{*}+\gamma_{c} S_{C} E_{T} \\
& +\left(\eta+\mu_{c}\right) I_{C}^{*}+\eta I_{C}+\left(\epsilon+\alpha_{b}\right) B_{I}^{*}+\nu I_{H T}+\mu_{e} E_{T}^{*}, \\
\mathcal{P}= & \beta_{T} \alpha_{p} P_{I} S_{H}+\beta_{T} \alpha_{b} B_{I} S_{H}+\theta S_{H} E_{T}+\mu_{h} S_{H}+\frac{\psi S_{H}^{*}}{S_{H}}+\mu_{h} I_{H T}+\frac{\beta_{T} \alpha_{p} P_{I} S_{H} I_{H T}^{*}}{I_{H T}} \\
& +\frac{\beta_{T} \alpha_{b} B_{I} S_{H} I_{H T}^{*}}{I_{H T}}+\left(\mu_{h}+\mu_{d}\right) I_{H C}+\frac{\theta S_{H} E_{T} I_{H C}^{*}}{I_{H C}}+\gamma_{p} S_{P} E_{T}+\left(\rho+\mu_{p}\right) S_{P} \\
& +\frac{\Lambda S_{P}^{*}}{S_{P}}+\left(\omega+\mu_{p}\right) I_{P}+\frac{\gamma_{p} S_{P} E_{T} I_{P}^{*}}{I_{P}}+\frac{\omega I_{P} P_{I}^{*}}{P_{I}}+\gamma_{c} S_{C} E_{T}+\left(\sigma+\mu_{c}\right) S_{C} \\
& +\frac{\phi S_{C}^{*}}{S_{C}}+\left(\delta+\alpha_{p}\right) P_{I}+\left(\eta+\mu_{c}\right) I_{C}+\frac{\gamma_{c} S_{C} E_{T} I_{C}^{*}}{I_{C}}+\left(\epsilon+\alpha_{b}\right) B_{I}+\frac{\eta I_{C} B_{I}^{*}}{B_{I}} \\
& +\mu_{e} E_{T}+\frac{v I_{H T} E_{T}^{*}}{E_{T}} .
\end{aligned}
$$


It can be seen from Eq. (26) that if $\mathcal{J}<\mathcal{P}$ then $\frac{d \mathcal{L}}{d t}<0$ and if $\Omega=\Omega^{*}$ then $\frac{d \mathcal{L}}{d t}=0$. Thus, the largest invariant set in $\Omega$ is the endemic equilibrium. Hence from the LaSalle invariant principle [17], we can conclude that, as $t \rightarrow \infty$, the solution of the model system (1) approaches the endemic equilibrium when $R_{0}>1$. Therefore, the endemic equilibrium is globally asymptotically stable in the invariant set $\Omega$ if $\mathcal{J}<\mathcal{P}$.

\section{Numerical simulations}

To understand well the dynamics of taeniasis and cysticercosis in humans, pigs and cattle, we simulate the model (1) using parameters from different literature and some are assumed as indicated in Table 2. To obtain initial conditions we consider a village with 5420 susceptible humans, 750 humans with taeniasis, 528 humans with cysticercosis, 1050 susceptible pigs, 620 infected pigs, 1250 susceptible cattle, 850 infected cattle and 1000 taenia eggs in the environment. We quantify pork and beef in terms of the number of infected pigs and cattle that are slaughtered for consumption. The Runge-Kutta order 4 numerical method is used to simulate the model in MATLAB software.

\subsection{The fourth order Runge-Kutta method (RK4)}

The 4th order Runge Kutta method (RK4) is a numerical method for solving a system of ordinary differential equations. The advantage of using RK4 over other numerical methods is that it is more accurate as it has high-order local truncation error $O\left(h^{4}\right)$ of the Taylor methods and eliminates the need of computing and evaluating the derivatives of a function. RK4 approximates the solution of the initial value system of the first order differential equation of the form

$$
\frac{d y}{d t}=f(t, y(t)) ; \quad y\left(t_{0}\right)=y_{0} .
$$

The method uses the initial value of the function to start the algorithm:

$$
\begin{aligned}
& k_{1}=h f\left(t_{i}, y_{i}\right), \quad k_{2}=h f\left(t_{i}+\frac{h}{2}, y_{i}+\frac{1}{2} k_{1},\right), \quad k_{3}=h f\left(t_{i}+\frac{h}{2}, y_{i}+\frac{1}{2} k_{2},\right), \\
& k_{4}=h f\left(t_{i+1}, y_{i}+k_{3},\right), \quad y_{i+1}=y_{i}+\frac{1}{6}\left(k_{1}+2 k_{2}+2 k_{3}+k_{4}\right),
\end{aligned}
$$

for each $i=1,2,3, \ldots, N-1$, where $h>0$ is the given step size and $k_{1}, k_{2}, k_{3}, k_{4}$ are constants that are used to eliminate the need for successive nesting in the second variable of $f(t, y)$ [3].

\subsection{Model simulation}

The infected cattle and pigs increase initially to their maximum in the first six months and later they decline and remain constant as illustrated in Fig. 3(a) and (b). The decline of infected cattle and pigs is in correspondence with the decline of taenia eggs in environment as shown in Fig. 3(d). This situation can happen if humans with taeniasis have toilets and hence do not shed taenia eggs in the environment. Susceptible cattle and pigs decrease to their lowest following infection by cysticercosis. However, they increase between the first and fourth year and thereafter remain constant.

In Fig. 3(c), susceptible humans decline rapidly in the first six months. However, at the end of the first year, susceptible humans increase gradually until the third year where they 

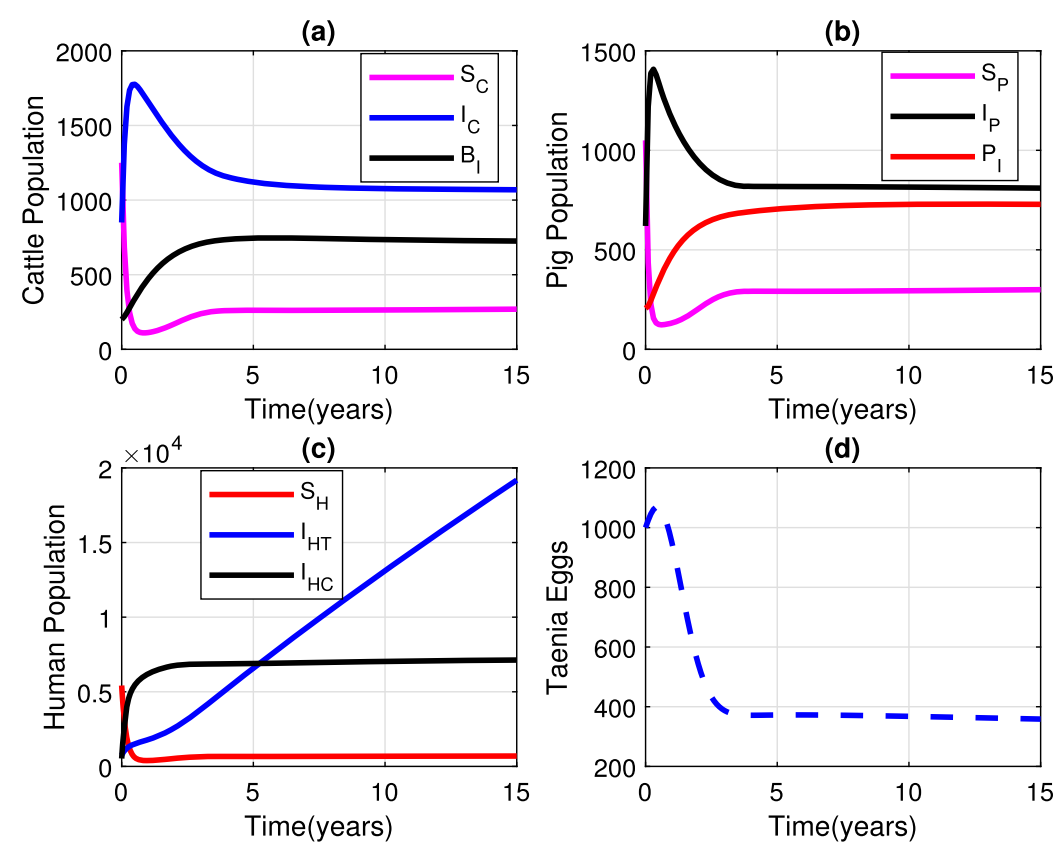

Figure 3 Dynamics of Humans, Pigs, Cattle and Taenia Eggs

remain constant. Humans with cysticercosis increase rapidly in the first six months and then with gradual increase until the second year where they remain constant. These trends are caused by variation of taenia eggs in the environment as depicted by Fig. 3(d) and consumption of raw or undercooked infected pork and beef. On the other hand, humans who are infected with taeniasis increase with time in the first two years and then increases linearly with time. This can happen if humans have habit of consuming raw or insufficiently cooked infected beef and pork.

\subsection{Effect of varying the most sensitive parameters}

In this subsection we present numerical simulation by considering the most sensitive parameters to observe how they affect disease transmission in humans, pigs and cattle. From the sensitivity analysis, the most positive sensitive parameters are the human recruitment rate $(\psi)$, probability of humans to be infected with taeniasis $\left(\beta_{T}\right)$ and the defection rate of humans with taeniasis $(v)$ whereas the most negative sensitive parameter is the natural mortality rate of humans $\left(\mu_{h}\right)$.

\subsubsection{Effect of varying human recruitment rate $(\psi)$}

The dynamics of taeniasis and cysticercosis shows that humans with taeniasis and cysticercosis, and infected cattle and pigs will increase in proportion to the human recruitment rate as illustrated in Fig. 4.

\subsubsection{Effect of varying defecation rate (v)}

Infected pigs and cattle, and humans who are infected with cysticercosis increase with time as a result of increase in defecation rate as depicted by Fig. 5(a), (b), (d). A different trend can be observed for humans who are infected with taeniasis in Fig. 5(c) where there is a 

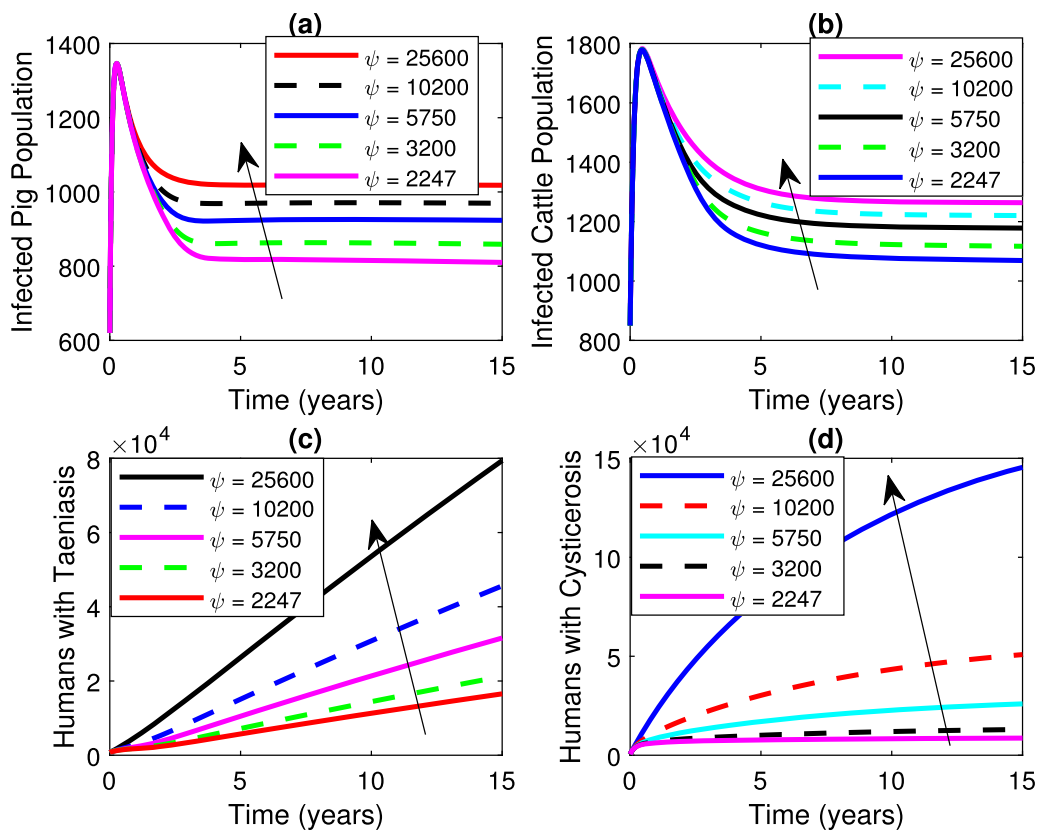

Figure 4 Impact of varying human recruitment rate on infected populations

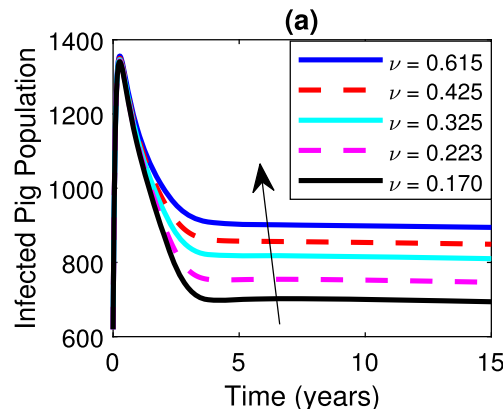

(c)

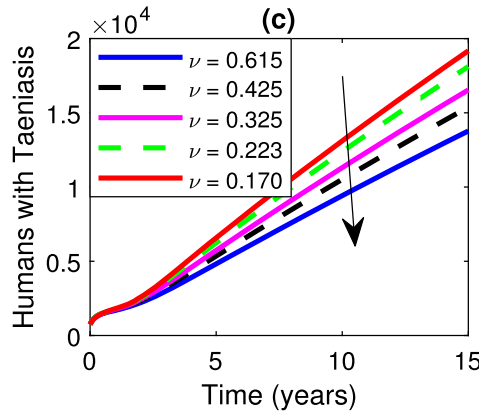

(b)

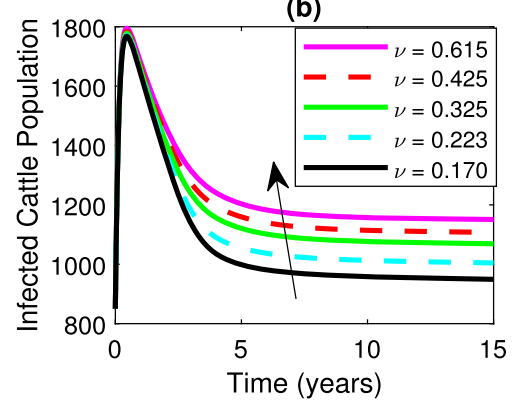

(d)

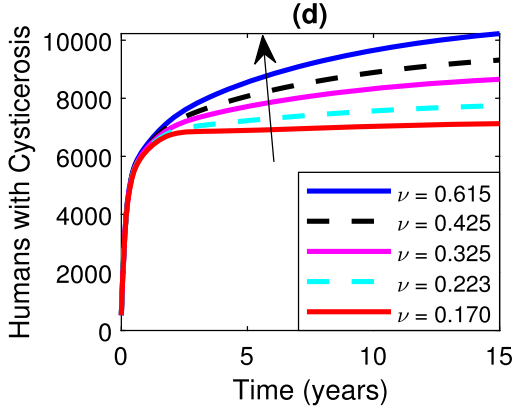

Figure 5 Impact of varying defecation rate on infected populations

decrease in cases of humans who are infected when the human defecation rate is increased. This is due to the fact that a high defecation rate can result in an expel of a high number of taenia eggs and so the possibility of recovery. 

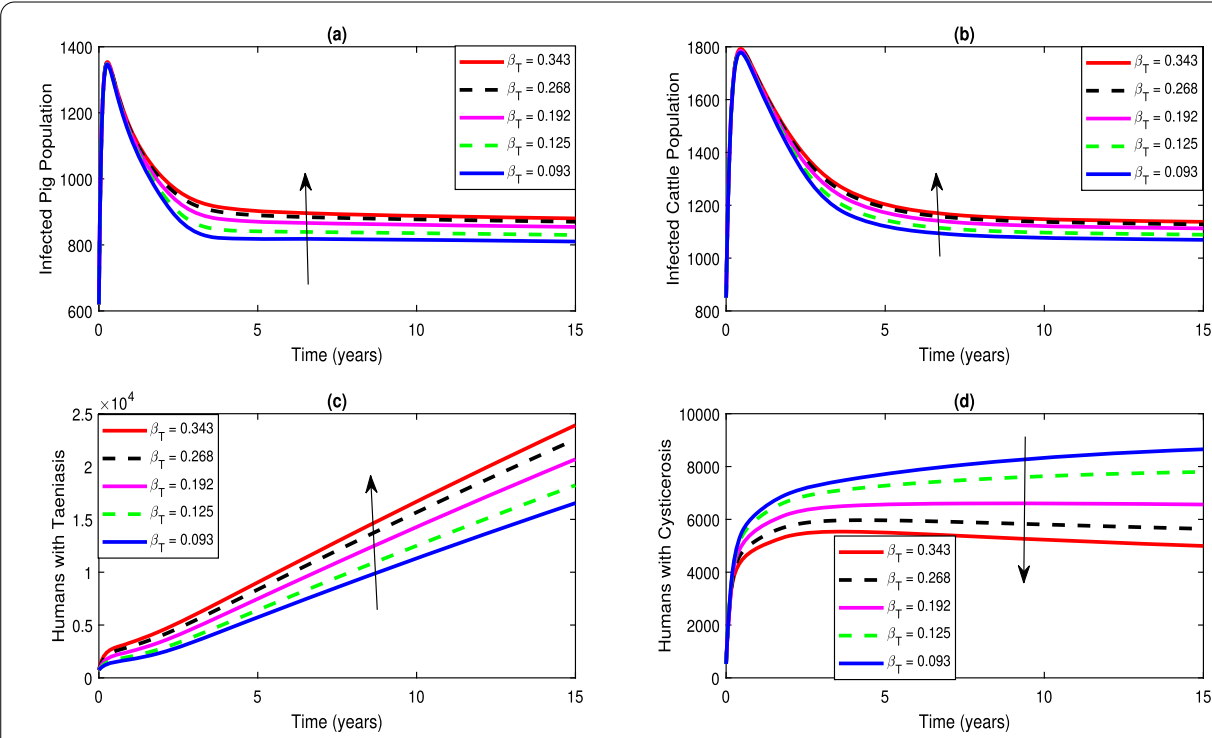

Figure 6 Impact of varying probability of infection on infected populations

\subsubsection{Effect of varying probability of taeniasis infection $\left(\beta_{T}\right)$}

In Fig. 6(a), (b), (c); the infected pigs, cattle and humans who are infected with taeniasis show an increase with time when the probability of taeniasis infection is increased. A different trend can be seen for humans who are infected with cysticercosis in Fig. 6(d), which shows the decrease in disease prevalence with increase in probability of taeniasis infection. This is because humans cysticercosis does not depend on the probability of human infection with taeniasis but on the rate at which humans consume T. solium eggs from the contaminated environment.

\subsubsection{Effect of varying human natural death rate $\left(\mu_{h}\right)$}

In Fig. 7, the results show that an increase in human natural mortality rate has negative impact in disease prevalence for all infected sub-populations. This indicates that human beings play an important role in transmission of the two diseases.

\section{Conclusion and recommendation}

In this paper, a mathematical model for the transmission dynamics of taeniasis and cysticercosis in humans, pigs and cattle is presented and analyzed. The model is well posed since the model solutions are positive and bounded. The disease free and endemic equilibria exist and their stability are investigated. The next generation approach is used to compute the basic reproduction number $R_{0}$. The analysis has shown that the disease free equilibrium is globally asymptotically stable when basic reproduction number $R_{0}<1$ while the endemic equilibrium is globally asymptotically stable when $R_{0}>1$. The effect of the most sensitive parameters in the diseases' transmission dynamics was assessed. Numerical results indicate that increasing human recruitment rate $(\psi)$ leads to an increased disease prevalence whereas increasing human natural death rate $\left(\mu_{h}\right)$ reduces disease prevalence in all populations. On the other hand, increasing probability of human infection with taeniasis $\left(\beta_{T}\right)$ increases the number of infected pigs, cattle and humans with taeniasis and reduces the number of cases for humans with cysticercosis. Similarly, increasing open hu- 

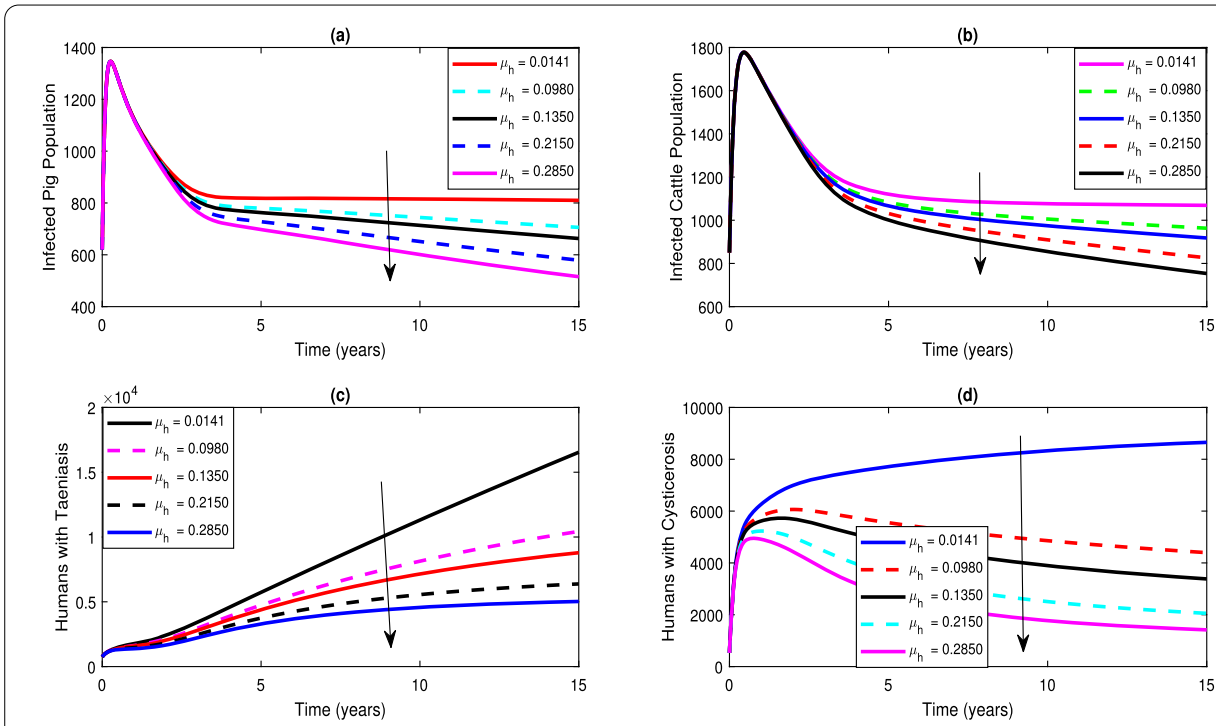

Figure 7 Impact of varying human natural death rate on infected populations

man defecation rate $(v)$ leads to an increased number of cases for infected pigs, cattle and humans with cysticercosis and decreases the number of cases for humans with taeniasis.

To control the diseases, the open human defecation rate should be reduced through use of toilets, especially in rural communities where pigs and cattle are kept under a free range system, treatment of infected individuals and proper cooking of pork and beef. To reduce the rate of transfer of infections from contaminated environment, we recommend pigs and cattle vaccination, indoor keeping of pigs and cattle as well as improvement in hygiene and sanitation. Moreover, infected pigs and cattle should be treated and meat inspection should be promoted to reduce the rate at which humans are infected with taeniasis.

\section{Acknowledgements}

The authors would like to thank the Ministry of Education, Science and Technology (MoEST) in Tanzania for supporting PhD studies and Mkwawa University College of Education (MUCE) for giving study leave to J.A. Mwasunda.

\section{Funding}

The Ministry of Education, Science and Technology (MoEST) supports the corresponding author's PhD studies at the Nelson Mandela African Institution of Science and Technology, Arusha, Tanzania.

Availability of data and materials

Most of data used in this research were found from different literature and some were assumed.

Competing interests

The authors declare that they have no competing interests.

\section{Authors' contributions}

JAM: Conceptualization, Model Formulation, Model Analysis and Drafting of the Manuscript; Jll: Model Formulation and Supervision; DK: Supervision; and DK: Supervision. All authors read and approved the final manuscript.

\section{Author details}

'Department of Applied Mathematics and Computational Science, The Nelson Mandela African Institution of Science and Technology, P.O. Box 447, Arusha, Tanzania. ${ }^{2}$ Mathematics Department, Mkwawa University College of Education, P.O. Box 2513, Iringa, Tanzania. ${ }^{3}$ Mathematics Department, Kabale University, P.O. Box 317, Kabale, Uganda.

\section{Publisher's Note}

Springer Nature remains neutral with regard to jurisdictional claims in published maps and institutional affiliations. 


\section{References}

1. Aung, A.K., Spelman, D.W.: Taenia solium taeniasis and cysticercosis in southeast Asia. Am. J. Trop. Med. Hyg. 94(5), 947-954 (2016)

2. Braae, U.C., Devleesschauwer, B., Gabriël, S., Dorny, P., Speybroeck, N., Magnussen, P., Torgerson, P., Johansen, M.V.: Cystisim—an agent-based model for taenia solium transmission and control. PLoS Negl. Trop. Dis. 10(12), 1-17 (2016)

3. Burden, R.L., Faires, J.D.: Numerical Analysis, Brooks. Cole, Belmont (1997)

4. Castillo-Chavez, C., Song, B.: Dynamical models of tuberculosis and their applications. Math. Biosci. Eng. 1(2), 361-404 (2004)

5. Chitnis, N., Hyman, J.M., Cushing, J.M.: Determining important parameters in the spread of malaria through the sensitivity analysis of a mathematical model. Bull. Math. Biol. 70(5), 1272-1296 (2008)

6. Cui, J., Wu, Z., Zhou, X.: Mathematical analysis of a cholera model with vaccination. J. Appl. Math. 2014, 1-17 (2014)

7. Del Brutto, O.H.: Human cysticercosis (taenia solium). Trop. Parasitol. 3(2), 100-103 (2013)

8. Dermauw, V., Dorny, P., Braae, U.C., Devleesschauwer, B., Robertson, L.J., Saratsis, A., Thomas, L.F.: Epidemiology of taenia saginata taeniosis/cysticercosis: a systematic review of the distribution in southern and eastern Africa. Parasites Vectors 11(1), 1-12 (2018)

9. Diekmann, O., Heesterbeek, J.A.P., Metz, J.A.: On the definition and the computation of the basic reproduction ratio $\mathrm{r} 0$ in models for infectious diseases in heterogeneous populations. J. Math. Biol. 28(4), 365-382 (1990)

10. Dumont, Y., Chiroleu, F., Domerg, C.: On a temporal model for the Chikungunya disease: modeling, theory and numerics. Math. Biosci. 213(1), 80-91 (2008)

11. Flisser, A., Rodríguez-Canul, R., Willingham, A.L. III: Control of the taeniosis/cysticercosis complex: future developments. Vet. Parasitol. 139(4), 283-292 (2006)

12. Gonzalez, A.E., Gilman, R.H., Garcia, H.H., Lopez, T.: Use of a simulation model to evaluate control programmes against taenia solium cysticercosis. In: Taenia Solium Cysticercosis: From Basic to Clinical Science, pp. 437-448. CAB International, Wallingford (2002)

13. Ito, A., Wandra, T., Yamasaki, H., Nakao, M., Sako, Y., Nakaya, K., Margono, S.S., Suroso, T., Gauci, C., Lightowlers, M.W.: Cysticercosis/taeniasis in Asia and the Pacific. Vector-Borne Zoonot. Dis. 4(2), 95-107 (2004)

14. José, M.V., Bobadilla, J.R., Sánchez-Torres, N.Y., Laclette, J.P.: Mathematical model of the life cycle of taenia-cysticercosis: transmission dynamics and chemotherapy (part 1). Theor. Biol. Med. Model. 15(1), 1-19 (2018)

15. Kavishe, M.D., Mkupasi, E.M., Komba, E.V., Ngowi, H.A.: Prevalence and risk factors associated with porcine cysticercosis transmission in Babati District, Tanzania. Livestock Res. Rural Dev. 29(1), 1-16 (2017)

16. Kyvsgaard, N.C., Johansen, M.V., Carabin, H.: Simulating transmission and control of taenia solium infections using a reed-frost stochastic model. Int. J. Parasitol. 37(5), 547-558 (2007)

17. LaSalle, J.P.: The Stability of Dynamical Systems, vol. 25. SIAM, Philadelphia (1976)

18. Lightowlers, M.W.: Eradication of taenia solium cysticercosis: a role for vaccination of pigs. Int. J. Parasitol. 40(10), 1183-1192 (2010)

19. Lightowlers, M.W., Rolfe, R., Gauci, C.G.: Taenia saginata: vaccination against cysticercosis in cattle with recombinant oncosphere antigens. Exp. Parasitol. 84(3), 330-338 (1996)

20. Lolika, P.O., Mushayabasa, S.: Dynamics and stability analysis of a brucellosis model with two discrete delays. Discrete Dyn. Nat. Soc. 2018,1-21 (2018)

21. Mkupasi, E., Kilemile, A., Mandike, O., Prosper, L., Ngowi, H.: Porcine cysticercosis—an emerging neglected food-borne parasitic zoonosis in urban settings in Tanzania: need for immediate control strategies. Tanzania Vet. J. 35(1), 112-117 (2018)

22. Mkupasi, E.M., Ngowi, H.A., Nonga, H.E.: Prevalence of extra-intestinal porcine helminth infections and assessment of sanitary conditions of pig slaughter slabs in Dar es Salaam city, Tanzania. Trop. Anim. Health Prod. 43(2), 417-423 (2011)

23. Mwanjali, G., Kihamia, C., Kakoko, D.V.C., Lekule, F., Ngowi, H., Johansen, M.V., Thamsborg, S.M., Willingham, A.L. III: Prevalence and risk factors associated with human taenia solium infections in Mbozi District, Mbeya Region, Tanzania. PLoS Negl. Trop. Dis. 7(3), 1-8 (2013)

24. Nyabadza, F., Aduamah, J.M., Mushanyu, J.: Modelling cholera transmission dynamics in the presence of limited resources. BMC Res. Notes 12(1), 1-8 (2019)

25. Okosun, K.O., Mukamuri, M., Makinde, D.O.: Global stability analysis and control of leptospirosis. Open Math. 14(1), 567-585 (2016)

26. Osman, S., Otoo, D., Sebil, C.: Analysis of listeriosis transmission dynamics with optimal control. Appl. Math. 11(7), 712-737 (2020)

27. Sánchez-Torres, N.Y., Bobadilla, J.R., Laclette, J.P., José, M.V.: How to eliminate taeniasis/cysticercosis: porcine vaccination and human chemotherapy (part 2). Theor. Biol. Med. Model. 16(1), 1-14 (2019)

28. Sun, G.-Q., Xie, J.-H., Huang, S.-H., Jin, Z., Li, M.-T., Liu, L.: Transmission dynamics of cholera: mathematical modeling and control strategies. Commun. Nonlinear Sci. Numer. Simul. 45, 235-244 (2017)

29. Symeonidou, I., Arsenopoulos, K., Tzilves, D., Soba, B., Gabriël, S., Papadopoulos, E.: Human taeniasis/cysticercosis: a potentially emerging parasitic disease in Europe. Ann. Gastroenterol. 31(4), 406-412 (2018)

30. Trevisan, C., Devleesschauwer, B., Schmidt, V., Winkler, A.S., Harrison, W., Johansen, M.V.: The societal cost of taenia solium cysticercosis in Tanzania. Acta Trop. 165, 141-154 (2017)

31. Van den Driessche, P., Watmough, J.: Reproduction numbers and sub-threshold endemic equilibria for compartmental models of disease transmission. Math. Biosci. 180(1-2), 29-48 (2002)

32. Wang, K., Zhang, X., Jin, Z., Ma, H., Teng, Z., Wang, L.: Modeling and analysis of the transmission of echinococcosis with application to Xinjiang Uygur autonomous region of China. J. Theor. Biol. 333, 78-90 (2013)

33. WHO: World health organization: epilepsy due to pork tapeworm: a serious public health problem in the united republic of tanzania (2018). https://www.who.int/taeniasis/en/

34. Winskill, P., Harrison, W.E., French, M.D., Dixon, M.A., Abela-Ridder, B., Basáñez, M.-G.: Assessing the impact of intervention strategies against taenia solium cysticercosis using the epicyst transmission model. Parasites Vectors 10(1), 1-14 (2017)

35. World Health Organization: WHO/FAO/OIE Guidelines for the Surveillance, Prevention and Control of Taeniosis/Cysticercosis. World Organisation for Animal Health, Paris (2005) 
36. World Health Organization: Taeniasis/cysticercosis fact sheet (2019). https://www.who.int/news-room/fact-sheets/detail/taeniasis-cysticercosis

37. Wu, L., Song, B., Du, W., Lou, J.: Mathematical modelling and control of echinococcus in Qinghai province, China. Math. Biosci. Eng. 10(2), 425-444 (2013)

38. Yang, Y., Feng, Z., Xu, D., Sandland, G.J., Minchella, D.J.: Evolution of host resistance to parasite infection in the snail-schistosome-human system. J. Math. Biol. 65(2), 201-236 (2012)

Submit your manuscript to a SpringerOpen ${ }^{\circ}$ journal and benefit from:

- Convenient online submission

- Rigorous peer review

- Open access: articles freely available online

- High visibility within the field

- Retaining the copyright to your article

Submit your next manuscript at $\boldsymbol{\Delta}$ springeropen.com 